\title{
Measurement of $\mathrm{CO}_{2}$ Concentration and Temperature in an Aero Engine Exhaust Plume Using Wavelength Modulation Spectroscopy
}

\author{
Thomas Benoy, David Wilson, Michael Lengden, Ian Armstrong, George Stewart, and Walter Johnstone
}

\begin{abstract}
In this paper, the 2f/1f tunable diode laser wavelength modulation spectroscopy is used for the simultaneous measurement of concentration and temperature of $\mathrm{CO}_{2}$ in the exhaust plume of an aero engine. The suitability of the $\mathrm{R} 48$ spectral feature of $\mathrm{CO}_{2}$ at $1997.2 \mathrm{~nm}$ is discussed for this project and for further application of $\mathrm{CO}_{2}$ tomographic imaging on large-scale aero-engines. To ensure accurate recovery of gas parameters at the high exhaust temperatures, a full spectral characterization of the absorption feature is presented using a direct spectroscopy. The $2 \mathrm{f} / \mathbf{1 f}$ method is validated in the laboratory for controlled gas mixtures and temperatures to recover concentration and temperature. Good agreement with the actual temperature and concentration values is demonstrated. Finally, single path measurements are presented for an aero engine exhaust showing good correlation with the measured engine conditions.
\end{abstract}

Index Terms-Optical sensor, high temperature spectroscopy, aerospace engineering, wavelength modulation spectroscopy, optical tomography.

\section{INTRODUCTION}

$\mathbf{T}$ HE fiber Laser Imaging of Turbine Exhaust Species (FLITES) project is a collaborative project between university partners Edinburgh, Manchester, Southampton and Strathclyde and several companies including Rolls-Royce, Shell and the Instituto Nacional Técnica Aeroespacial (INTA). The project's aim is to measure gaseous and particulate species concentration in a two-dimensional plane in the exhaust plume of large commercial aero engines [1].

One of the main deliverables in this project is the production of a cross-sectional, 2-dimensional tomographic image of $\mathrm{CO}_{2}$ concentration using 126 individual beams paths directed through the engine exhaust plume. This ability to image the distribution of $\mathrm{CO}_{2}$ will reduce engine testing time and

Manuscript received July 27, 2017; revised August 2, 2017; accepted August 3, 2017. Date of publication August 11, 2017; date of current version September 8, 2017. This work was supported by the U.K. Engineering and Physical Sciences Research Council in the context of Fiber-Laser Imaging of Gas Turbine Exhaust Species Project under Grant EP/J002178/1. The associate editor coordinating the review of this paper and approving it for publication was Prof. Tarikul Islam. (Corresponding author: David Wilson.)

T. Benoy was with the Electronic and Electrical Engineering Department, University of Strathclyde, Glasgow G1 1XW, U.K. He is now with the Cochin University of Science and Technology, Kochi 682022, India.

D. Wilson, M. Lengden, G. Stewart, and W. Johnstone are with the Electronic and Electrical Engineering Department, University of Strathclyde, Glasgow G11XW, U.K. (e-mail: david.wilson.102@strath.ac.uk; michael. lengden@strath.ac.uk; g.stewart@strath.ac.uk; w.johnstone@strath.ac.uk).

I. Armstrong was with the Electronic and Electrical Engineering Department, University of Strathclyde, Glasgow G1 1XW, U.K. He is now with OptoSci Ltd., Glasgow G32 8NB, U.K.

Digital Object Identifier 10.1109/JSEN.2017.2738333 commissioning costs, and provide valuable real-time information to engine manufacturers and operators. For example, a real-time imaging approach will highlight the variability in the individual burner performance in the engine, providing earlier indication of burner maintenance requirements and reducing risk of engine failure. In addition, the current methodology for ascertaining the $\mathrm{CO}_{2}$ emission of a particular engine type, and ensuring it fulfils the necessary regulatory requirements, requires intrusive, extractive sampling of the exhaust at multiple locations. This implies that engines under test are operational over significantly longer periods than would be necessary with real time imaging methodologies.

The technique of tunable diode laser spectroscopy with wavelength modulation (TDLS-WM) is ideally suited for this imaging application, as it has a proven record of non-invasive, in situ measurement in harsh environments [2]-[5]. Recent developments have reduced intensity fluctuation noise prevalent in high temperature combustion environments [5]-[7] and have improved the accuracy of this high frequency modulation technique, providing measurements that require no further intensity calibration.

In this paper, we present the validation of the $\mathrm{CO}_{2}$ concentration measurement system designed for the FLITES project. This has been achieved by measuring the path-integrated absorbance from the exhaust of a commercial aero engine for a single beam path using the $2 \mathrm{f} / 1 \mathrm{f}$ technique developed by Rieker et al. [7]. Values of the average plume temperature and the gas concentration are then extracted from the recovered $2 \mathrm{f} / 1 \mathrm{f}$ signals. To ensure the accuracy of engine measurements, a full laboratory validation of the $2 \mathrm{f} / 1 \mathrm{f}$ technique was carried out and the temperature dependence of the spectral parameters for the targeted absorption feature were measured using a high temperature spectrometer. This was achieved by using a least squares fitting algorithm to compare experimentally obtained data in a controlled environment with modelled spectra, where two of the modelling parameters are varied until the total least squares (LSQ) value between the model and the experimental data has reached a minimum.

Section II provides a justification of the spectral feature selected for aero engine measurements and presents measurements of the temperature dependence of collisional broadening parameters and the line-strength of the chosen spectral feature. Section III discusses the requirement for significant laser characterization and post-signal processing for the 2f/1f methodology. It goes on to provide further 
justification of the technique for this target application, including a discussion of data transmission and storage limitations. Section III describes how average temperature and the path integrated concentration are obtained from a single $\mathrm{CO}_{2}$ spectral feature. Single channel $2 \mathrm{f} / 1 \mathrm{f}$ measurements of $\mathrm{CO}_{2}$ concentration and average plume temperature in the exhaust of a commercial, turbofan engine with a bypass are presented in Section IV for varying engine thrust. Again the LSQ methodology as described above is used with concentration and temperature as the variables. A high degree of correlation with the measured engine core temperature is demonstrated.

\section{Spectral Feature Validation}

\section{A. Spectral Feature Selection}

Although this paper only presents path integrated $\mathrm{CO}_{2}$ concentration data for a single beam path and on a smaller engine type to the one proposed for the tomographic imaging system, consideration must be given to the scheme proposed for the FLITES project [1]. To achieve the necessary optical power for each of the 126 beams, and allow for easy signal distribution using optical fiber couplers, wavelength regions for consideration must allow the use of high power optical fiber amplifiers and optical fiber components. This limits wavelength selection to wavelengths around $1550 \mathrm{~nm}$ and $2000 \mathrm{~nm}$ using either an erbium doped fiber amplifier or a thulium doped fiber amplifier respectively. $\mathrm{CO}_{2}$ absorption occurs in both these wavelength regions. However, the increased probability of absorption for the $2000 \mathrm{~nm}$ band makes this region significantly more attractive for measurement in harsh environments, where a high SNR is targeted to ensure accurate measurement.

The secondary criterion for spectral feature selection is its isolation from spectral features of other gases, particularly water vapour. This requires consideration of the gas-interaction path lengths in the engine plume and the surrounding environment. Each launch to receive path length for the finalised tomographic system is $7 \mathrm{~m}$, and a detailed description of the optics has been presented previously [8].

For the engine used in this paper the estimated plume diameter is $80 \mathrm{~cm}$ and the launch to receive distance is approximately $2 \mathrm{~m}$, the plume temperature is assumed to be $200{ }^{\circ} \mathrm{C}$ and the $\mathrm{CO}_{2}$ concentration is between $1-3 \%$. Figure 1 is a spectral model of $\mathrm{CO}_{2}$ and water vapour for these conditions, plotted using the HITRAN2012 database [9]. The only other species that are significantly infrared active in this wavelength region are output at such low concentrations that they do not require further consideration. As can be seen, the most isolated $\mathrm{CO}_{2}$ spectral feature is at a wavelength of $\sim 1997.2 \mathrm{~nm}$, which is the $\mathrm{R}(48)_{e}$ component of the $\mathrm{v}_{1}+2 \mathrm{v}_{2}+v_{3}$ band.

An Eblana Photonics Multi Quantum Well Distributed Feedback (DFB) laser (EP1997-DMB01-FM) was chosen to access this wavelength, which is within the amplification range of a thulium doped fiber amplifier designed and manufactured by Feng et al. at the University of Southampton as part of the FLITES project [10].

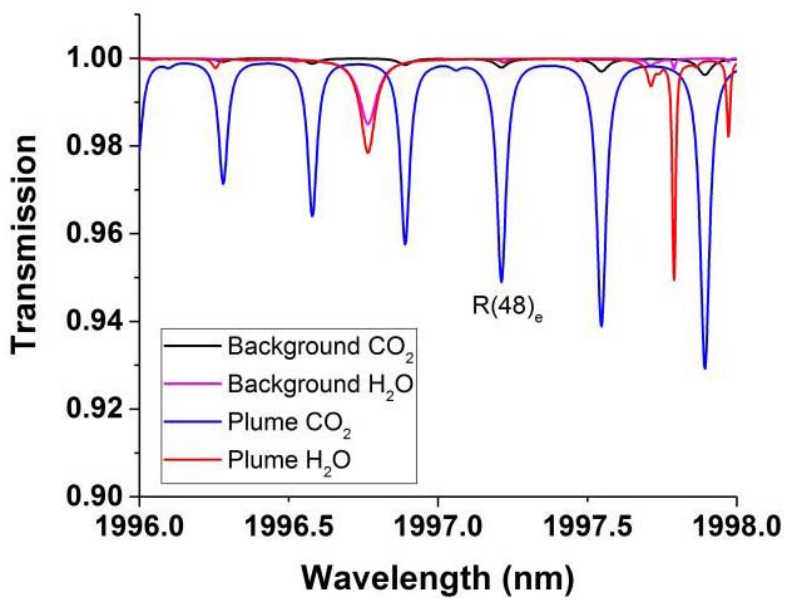

Fig. 1. Modelled spectra of the background and plume $\mathrm{CO}_{2}$ and water vapour. The plume concentration is assumed to be $3 \%$ for $\mathrm{CO}_{2}$ and $5 \%$ for $\mathrm{H}_{2} \mathrm{O}$ and the temperature is $200{ }^{\circ} \mathrm{C}$, the background concentration is $0.04 \%$ for $\mathrm{CO}_{2}$ and $1 \%$ for $\mathrm{H}_{2} \mathrm{O}$ and the temperature is $15^{\circ} \mathrm{C}$.

\section{B. High Temperature Spectroscopic Measurements}

The HITRAN [9], GEISA [11] and HITEMP CDSD [12] databases provide data for the relevant parameters required to model spectral features of $\mathrm{CO}_{2}$, including the line-strength, collisional self- and air-broadening parameters, wavelength pressure shifts and partition functions. However, there is limited information on the temperature dependence of collisional broadening parameters. In fact, the GEISA, HITRAN and the standard HITEMP databases only provide data on the temperature dependence of the air-broadening parameter. This limits their use for high temperature applications in harsh environments and leaves only a single database source for temperature dependence modelling of $\mathrm{CO}_{2}$ - HITEMP CDSD. A number of experimental studies have also been carried out on the spectral features of $\mathrm{CO}_{2}$ around 2000nm [13]-[15]. Webber et al. [15] provide data for line-strength and selfbroadening co-efficient for the R(48) transition. However, none of the experimental studies have data for the temperature dependence of all the necessary parameters. Therefore, a full investigation was carried out to measure these parameters using the high temperature cell detailed below and shown in Figure 2.

The cell operates at temperatures up to $750{ }^{\circ} \mathrm{C}$ and from vacuum pressure to $\sim 3$ bars. The inner cell (A), called the gas cell, is constructed entirely from fused silica and is used to hold the gas mixture of interest. The gas cell is contained within a much larger outer cell (B) constructed from 316 stainless steel.

The outer cell is a 4-way-cross chamber, $310.8 \mathrm{~mm}$ in length with an inner diameter of $146.4 \mathrm{~mm}$ and space for four CF-160 flanges. One of the CF-160 flanges (C) is further split into a central CF-40 flange, containing two Swagelok 1/4" stainless steel pipe feed-throughs (G), and six CF-16 flanges used for thermocouple and electrical feedthroughs. Two further flanges, located opposite each other (D \& E), are designed to contain windows at Brewster's angle. The main window housing was constructed from 316 stainless steel by CaburnMDC, and each housing contains a $4 \mathrm{~mm}$ thick, 


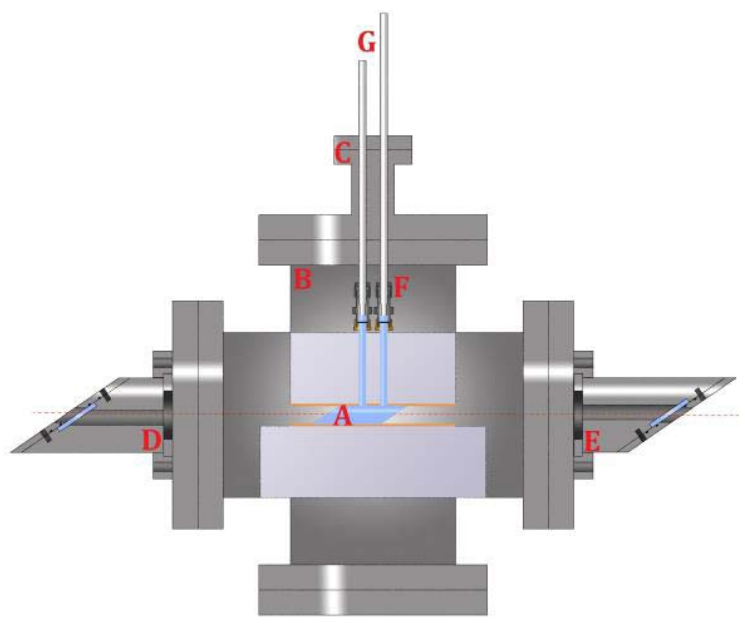

Fig. 2. High temperature gas cell used to fully characterize the temperature dependence of the R(48) spectral feature and to carry out all the TDLS-WM measurements in the laboratory.

40mm diameter Spectrosil window manufactured by Crystran Ltd.

The gas cell is $55 \mathrm{~mm}$ long and $16 \mathrm{~mm}$ diameter, with $2 \mathrm{~mm}$ thick end windows set to Brewster's angle, and is surrounded by a $2 \mathrm{~mm}$ thick, $150 \mathrm{~mm}$ long ceramic cylinder. Two lengths of RW-80 resistance wire are wrapped around the ceramic cylinder to provide uniform heating to the cell, and the temperature is controlled using two Fuji temperature controllers and solid state relays, with feedback from two thermocouples located at the cell windows. Rigidised Insulfrax $(\mathrm{H})$ is used to insulate the gas cell and surrounding ceramic and also provide support for the gas cell's two glass arms. Gas passes through the cell via two $75 \mathrm{~mm}$ long glass access tubes that are attached to $1 / 4$ " stainless steel piping using Swagelok Ultra-Torr seals, $(\mathrm{F})$. All pipework prior to the gas cell is heated using either RW-80 resistance wire or heat-tape.

The rest of the experimental set-up is shown in figure 3 . It should be noted that the high frequency signal generator, the lock-in amplifiers (LIAs) and the bias-T are not used for the fundamental spectroscopic measurements. They are included in fig. 3 to show the full experimental set-up for the TDLS-WM validation work in Section III.

For the spectral characterization work, the laser was thermally tuned to the centre wavelength of the $\mathrm{R}(48)_{\mathrm{e}}$ transition using a Thorlabs TED200C temperature controller. A $100 \mathrm{~Hz}$ sawtooth current ramp, with an amplitude of $80 \mathrm{~mA}$, was then applied to the laser via a Thorlabs LDC210 laser current controller to repeatedly scan the output wavelength over the targeted spectral line. This provided wavelength tuning of the order of $9 \mathrm{GHz}$, which is approximately three times larger than the full width half maximum (FWHM) of the absorption feature. The laser was set to operate below lasing threshold for the first $5 \%$ of the sawtooth ramp, providing an offset signal for determining dark-current and thermal drift.

The output from the laser passed through a $1 \times 250: 50$ optical fiber coupler. One output arm of the coupler was connected to a fiber ring resonator, with an FSR of $0.1238 \mathrm{GHz}$, providing a relative wavelength tuning measurement [16]. The output from the second coupler arm passed through an
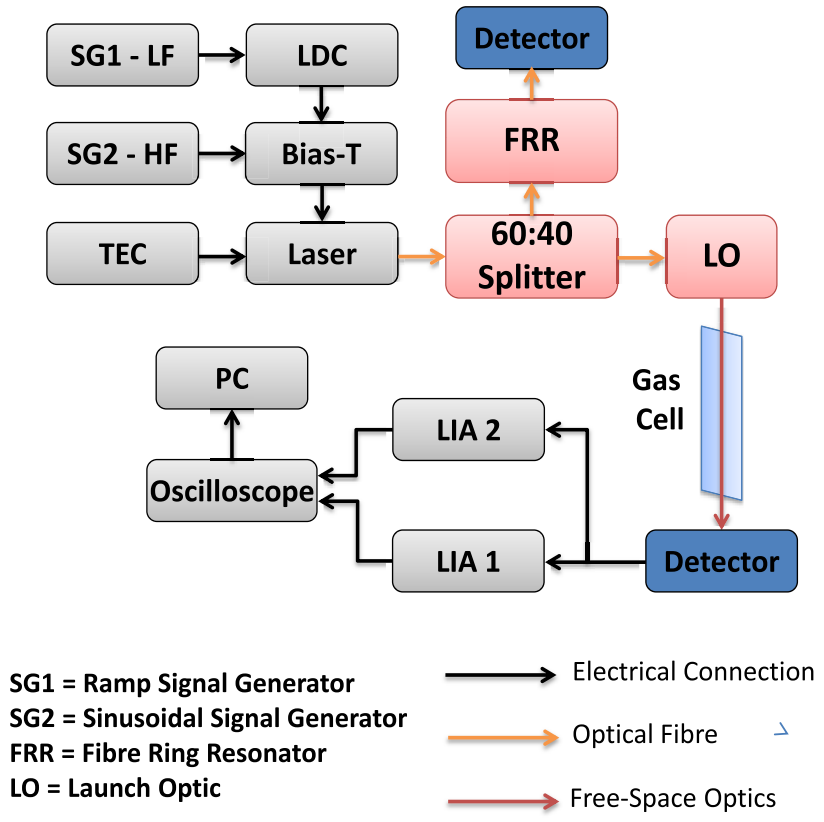

Fig. 3. Experimental setup for the fundamental spectroscopic and wavelength modulation spectroscopy measurements. For the fundamental measurements the modulation signal generator, the bias- $T$ and the lock-in amplifiers are not used.

aspheric collimation lens (Thorlabs F028APC-2000) and was then directed through the gas cell via the Brewster angled windows. Extended InGaAs detectors are used for both optical signals; the resonator output falls incident onto a Thorlabs PDA10D-EC and the output from the gas cell onto a Thorlabs DET10D/M. The photodiode outputs were monitored using a TDS3014B Tektronix oscilloscope, with an 8-bit vertical resolution, and the data was transferred to a PC using a GPIB controlled LabVIEW Program.

The obtained spectra were modelled using the Beer-Lambert law

$$
I_{\text {out }}=I_{\text {in }} e^{-k(\nu, T) P_{\text {tot }} \chi_{n} l}
$$

where $I_{\text {in }}$ is the incident light intensity on the volume of gas, $k(v, T)$ is the absorption coefficient at a wavenumber $v$, $P_{t o t}$ is the total pressure, $\chi_{n}$ is the mole fraction of species $n$ and $l$ is the gas cell length. $k(v, T)$ can be expressed in terms of the temperature dependent transitional line-strength, $S(T)$, and the transitional line-shape, $\phi$,

$$
k(v, T)=S(T) \phi\left(v-v_{0}\right)
$$

where the total area of $\phi$ has been normalised to 1 .

Typically, the line-shape is described by a Voigt profile, which is the convolution of a Gaussian, $\phi_{g}$, and a Lorentzian profile, $\phi_{L}$, representing Doppler and collisional broadening respectively. It has been assumed that for a given measured cell temperature the Doppler broadening term can be accurately calculated, and is therefore not included as a fitting parameter in the LSQ algorithm.

The Lorentzian profile FWHM used to describe the collisional broadening for each species is given as

$$
\Gamma_{l}=P_{t o t} \sum_{n} \chi_{n} \Gamma_{l}^{n}\left(T_{0}\right)\left(\frac{T_{0}}{T}\right)^{m_{n}}
$$




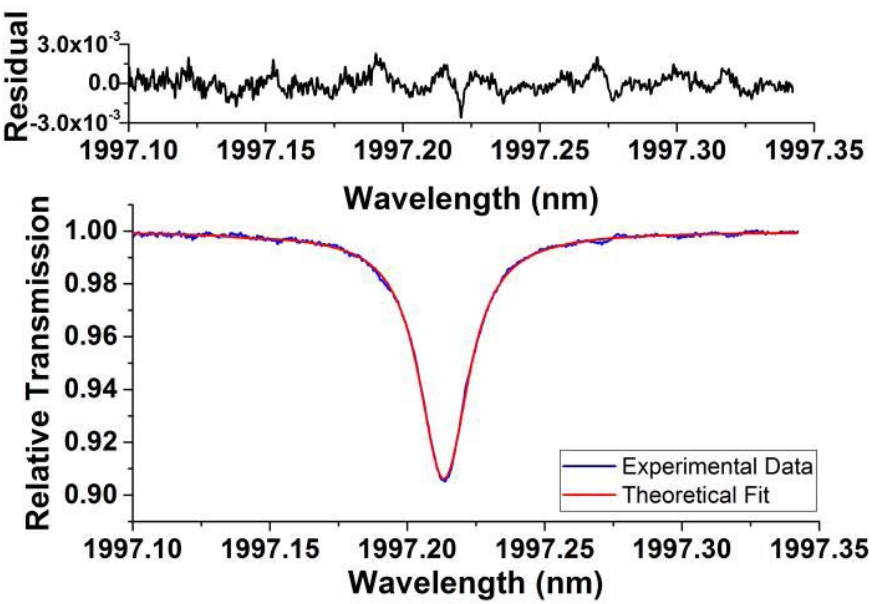

Fig. 4. Normalized absorption feature fitted against HITRAN model (theoretical fit red experimental data blue), residual difference between model and experimental data is shown above.

where $\Gamma_{L}^{n}\left(T_{0}\right)$ is the broadening parameter for each species, $n$, at the reference temperature $T_{0}=296 \mathrm{~K}$ and $m_{n}$ is the temperature exponent describing the variation of broadening as a function of temperature for each individual species.

As evidenced above, the integrated area of the spectral feature and the absorption line shape are affected by multiple parameters including concentration, pressure, temperature, and the nature of the collision partner gas. This work focuses on the temperature dependence of the line-strength and also the collisional broadening of $\mathrm{CO}_{2}-\mathrm{CO}_{2}$ and $\mathrm{CO}_{2}$-air interactions, as these are the most likely collisional partners that will be found in an exhaust plume.

The $\mathrm{CO}_{2}-\mathrm{CO}_{2}$ collisional broadening parameter and its temperature dependence were obtained by measuring the $\mathrm{R}(48)$ spectral feature for gas samples of $100 \% \mathrm{CO}_{2}$, at pressures ranging from 50 mbar to 500 mbar in 50 mbar increments, measured using an MKS Baratron Manometer (626C) with a quoted accuracy of $0.25 \%$ of reading. These measurements were repeated for temperatures ranging from $50^{\circ} \mathrm{C}$ up to $550^{\circ} \mathrm{C}$ in $50^{\circ} \mathrm{C}$ increments, with a measured temperature variation of $\pm 1.7 \%$ across the gas cell. Theoretical Voigt profiles were modelled using the HITRAN2012 database and the least squares fitting procedure described in Section I was used with the experimentally obtained data. In this case, the line-strength and the self-broadening parameter were the variables in the model and a typical fit shown in figure 4 .

For each temperature increment the self-broadening parameters obtained from the LSQ procedure were plotted as a function of pressure, and a linear fit was carried out to provide self-broadening co-efficients in the form

$$
\frac{\Gamma_{l}^{C O_{2}}}{P_{t o t}}=\Gamma_{l}^{C O_{2}}(296)\left(\frac{T_{0}}{T}\right)^{m_{C O}}
$$

as shown in figure 5 .

To calculate the temperature dependence of the broadening parameters a linear fit of $\log \left(\Gamma_{L}(T)\right) / P_{t o t}$ versus $\log \left(T_{0} / T\right)$ is carried out, as shown in Figure 6. The gradient of this linear fit is the temperature dependence of the self-broadening parameter, $m_{\mathrm{CO}_{2}}$, and the intercept with the y-axis provides

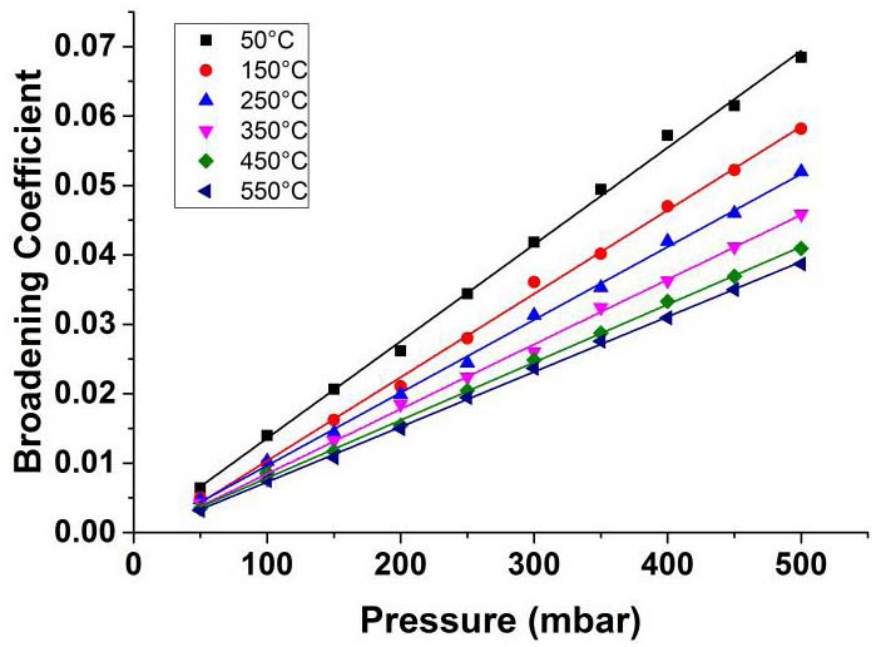

Fig. 5. Linear fit for the self-collisional broadening parameter for temperatures ranging from $50^{\circ}$ to $550^{\circ}$.

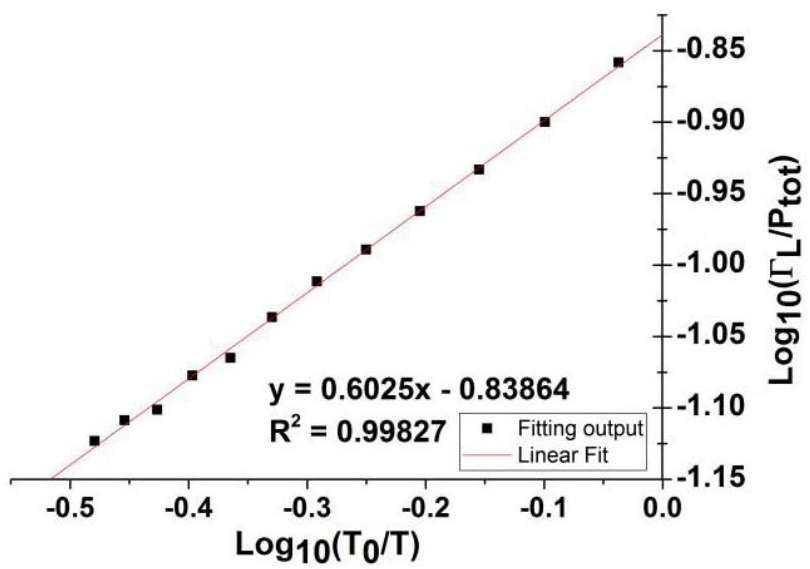

Fig. 6. Linear fit for the fitting output of logarithmic broadening parameter versus the logarithmic temperature ratio.

the broadening value at the reference temperature $\Gamma_{L}^{C O 2}(296)$. The newly fitted values of line-strength, $\Gamma_{L}^{C O 2}(296)$ and $m_{\mathrm{CO}_{2}}$ are then added as constants to the spectral model to allow the calculation of the temperature dependence of the aircollisional broadening term. This was achieved by repeating the experimental measurements using gas mixtures of $75 \%$, $50 \%$ and $30 \% \mathrm{CO}_{2}$ in air, and using $\Gamma_{l}^{a i r}$ as the variable in the LSQ fitting procedure. A comparison of these measured values and other published values are shown in table 1.

\section{WaVelength Modulation Spectroscopy}

\section{A. Considerations for Harsh Environments}

The proposed $\mathrm{CO}_{2}$ measurement methodology for the FLITES project is the $1^{\text {st }}$ harmonic normalized $2^{\text {nd }}$ harmonic technique (2f/1f) that has been extensively researched by the Hanson Group at Stanford University [6]. This technique has a number of advantages over other TDLS techniques. Firstly, it doesn't require a non-absorbing off-line background region for normalization. Secondly, there is removal of optical noise through the normalization procedure. The final output from 
TABLE I

Calculated Values of the Air- and Self-Broadening CO-EFFICIENTS OF $\mathrm{CO}_{2}$ AND VALUES FOR THEIR TEMPERATURE DEPENDENCE AND A COMPARISON WITH VALUES FROM A NUMBER OF DATABASES

\begin{tabular}{cccccc}
\hline & $\begin{array}{c}\text { This } \\
\text { Work }\end{array}$ & $\begin{array}{c}\text { HITRAN } \\
2012\end{array}$ & $\begin{array}{c}\text { CDSD- } \\
\text { HITEMP }\end{array}$ & GEISA & $\begin{array}{c}\text { Webber } \\
\text { et.al.[15] }\end{array}$ \\
\hline$\gamma_{\text {self }}$ & 0.073 & 0.073 & 0.0814 & 0.745 & 0.0753 \\
$\gamma_{\text {air }}$ & 0.063 & 0.0673 & 0.0687 & 0.0673 & No data
\end{tabular}

$\begin{array}{llll}m_{\text {self }} & 0.613 \quad \text { No data } & 0.64 \quad \text { No data No data }\end{array}$

$\begin{array}{lllll}m_{\text {air }} & 0.73 & 0.74 & 0.71 & 0.74 \quad \text { No data }\end{array}$

the $2 \mathrm{f} / 1 \mathrm{f}$ technique is given by the equation

$$
\frac{2 f}{1 f}=\sqrt{\left(\frac{X_{2 f}}{R_{1 f}}-\frac{X_{2 f}^{b k}}{R_{1 f}^{b k}}\right)^{2}+\left(\frac{Y_{2 f}}{R_{1 f}}-\frac{Y_{2 f}^{b k}}{R_{1 f}^{b k}}\right)^{2}}
$$

where $X_{2 f}$ and $Y_{2 f}$ are the second harmonic in-phase and quadrature outputs from a lock-in amplifier (LIA) and $R_{1 f}$ is the magnitude of the LIA first harmonic output during lasergas interaction, $X_{2 f}^{b k}$ and $Y_{2 f}^{b k}$ are the LIA second harmonic outputs and $R_{1 f}^{b k}$ is the magnitude of the LIA first harmonic output when no gas interaction has occurred.

Using the spectral data reported in section II allows accurate spectral modelling of the $2 \mathrm{f} / 1 \mathrm{f}$ signals. However, a number of other considerations need to be taken into account, particularly for the case of the FLITES project. These include the expected high noise levels in the exhaust plume, the effects of the laser source characteristics on the $2 \mathrm{f} / 1 \mathrm{f}$ technique, the introduction of a thulium doped fiber amplifier to the measurement system and the significant data processing required for 126 simultaneous measurements.

Typically, 2f/1f spectra are analysed using the methodology described by Sun et al. [17], where the entire modulated experimental signal is recorded and processed using a software LIA that is also used to demodulate a simulated WMS signal. This allows the same LIA bandwidth to be applied to both the theoretical and experimental data. For the FLITES project this would require $2 \mathrm{MS} \mathrm{s}^{-1}$ data recording per channel to achieve 10 samples per sine wave period. With the requirement for 126 channels to allow tomographic imaging this would imply at least $1 \mathrm{~Tb}$ of data being recorded for 30 minutes of aero-engine operation. As engines typically operate for over six hours per day this implies that the required data transmission and storage rates are far higher than realistically achievable. In the final tomography campaign the transmission rates and the total recorded data will be reduced by demodulating the 126 2f/1f signals at source using custom FPGA designs [18]. The in-phase and quadrature first and second harmonic signal measurements will then be transferred to a PC and recorded, reducing the data rate to $10 \mathrm{kSs}^{-1}$ per channel. This approach requires the signal analysis to be carried out using the WMS Fourier model [5], where the individual harmonic signals are modelled using pre-characterized laser modulation parameters. As this paper aims to replicate as closely as possible the final system for FLITES, the Fourier methodology has also been used in this work.

The laser modulation parameters required for the Fourier model are $i_{0}$ and $i_{2}$ - the ratio of the linear intensity modulation amplitude and the non-linear intensity modulation amplitude with respect to the laser intensity, $\psi_{1}$ and $\psi_{2}$ - the phase differences between the frequency modulation and the linear and non-linear intensity modulation respectively, and $\delta v$ - the wavelength modulation amplitude.

As shown previously [19], the Eblana laser used in this work exhibits a high degree of non-linearity in its output intensity response, and significant phase variation between its instantaneous intensity modulation (AM) and its frequency modulation (FM) as a function of drive current. Further non-linearity in the laser intensity occurs during optical amplification using the TDFA and via optical components that have significant wavelength dependence, such as fiber couplers. This non-linear AM signal is not removed through the division of the second harmonic magnitude by the first harmonic magnitude and is therefore removed using background subtraction, as described in equation 7. As the output characteristics of the TDFA are expected to drift during long-term operation it is essential that the intensity modulation characteristics of the optical source are monitored in real-time and in situ.

Another important consideration in the $2 \mathrm{f} / 1 \mathrm{f}$ technique is the measurement of absolute wavelength. The relative wavelength scale was measured using the fiber ring resonator described in Section II. In direct TDLS the assignment of the absolute wavelength is obtained by using a feature in the experimentally recovered absorption spectrum, typically the peak absorption. However, in the $2 \mathrm{f} / 1 \mathrm{f}$ model this peak position varies as a function of the modulation index, $\mathrm{m}=\delta \nu / \gamma$. Furthermore, the laser centre wavelength shifts during high frequency modulation due to second order current heating effects. The assigned centre wavelength therefore has to be included as a fitting parameter in the spectral modelling to improve accuracy.

\section{B. Validation of $2 f / 1 f$ in the Laboratory}

For the laboratory validation of the 2f/1f technique the experimental system shown in figure 3 was used. However, in this case the detector output from the measurement arm of the 50:50 splitter was connected to two Perkin-Elmer 7280 LIAs. One of the LIAs is used to output the magnitude of the first harmonic signal $\left(R_{1 f}\right)$ and the second to output the second harmonic signal in phase $\left(\mathrm{X}_{2 \mathrm{f}}\right)$ and at quadrature $\left(\mathrm{Y}_{2 \mathrm{f}}\right)$. A $5 \mathrm{~Hz}, 70 \mathrm{~mA}_{\mathrm{pp}}$ saw-tooth current ramp and a $200 \mathrm{kHz}$ sinusoidal current modulation were applied to the laser simultaneously via a bias-T. A $200 \mathrm{kHz}$ modulation frequency was chosen to ensure that operation was outside the expected noise spectrum of the aero-engine but still within the bandwidth of the proposed detection electronics designed for the FLITES project [20]. 


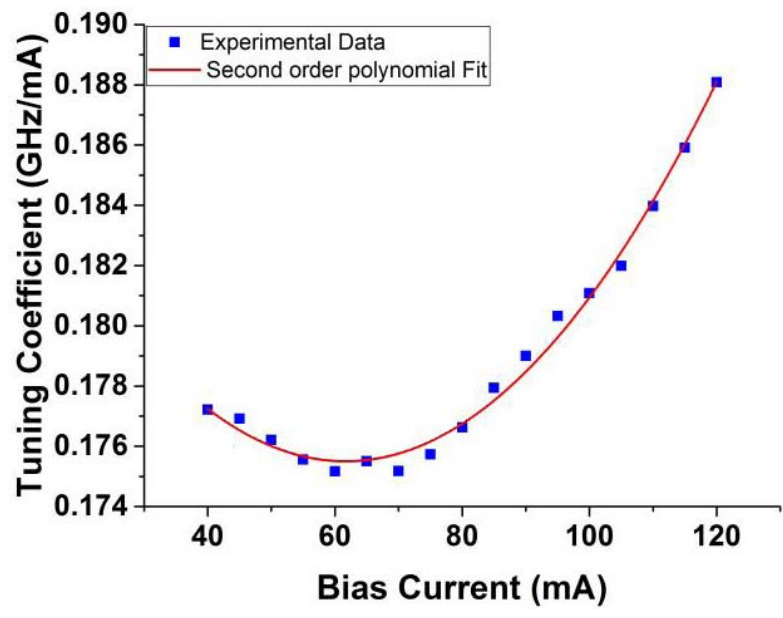

Fig. 7. Measurement of the tuning co-efficient as a function of the lasers bias current.

The values of $i_{0}$ and $i_{2}$ were measured through the gas cell containing $100 \%$ nitrogen, providing compensation for any static wavelength dependent transmission variation due to optical components in the cell, as well as in the optical fiber components. The lasers tuning co-efficient (shown in figure 7) and the phase terms, $\psi_{1}$ and $\psi_{2}$, were measured as a function of laser bias-current, using the methodology described in a previous publication [19].

Figure 8 shows an experimentally obtained 2f/1f signal using a $15 \mathrm{~mA}$ current modulation amplitude and a temperature of $200^{\circ} \mathrm{C}$, which is the approximate temperature measured from the engine exhaust in section IV, and a gas concentration of $100 \%$, equivalent to a $6.25 \%$ plume concentration over $80 \mathrm{~cm}$. The figure also shows a modelled spectral fit for temperature and concentration using a least squares error algorithm in Matlab and the measured laser parameters. During this fitting process it was assumed that the pressure in the cell was constant and the temperature was uniform. This assumption is also made for the engine plume measurements, where a top-hat function of temperature is used over a plume diameter of $80 \mathrm{~cm}$ and the pressure was the measured atmospheric pressure.

As discussed previously [19], $\psi_{1}$ varies by $18 \%$ and $\delta v$ varies by $6 \%$ over the current scan used to interrogate the $\mathrm{R}(48)$ spectral feature, as shown in figure 7. A simulated 2f/1f signal for the expected aero-engine conditions was developed with added white Gaussian noise $(\mathrm{SNR}=5 \mathrm{~dB})$ that included the variation of both $\psi_{1}$ and $\delta v$. Concentration and temperature values were then calculated for this simulated data using the least squares fitting algorithm and three different models; one that had a constant value of $\psi_{1}$ and varying $\delta v$, one that included a constant value of $\delta v$ and varied $\psi_{1}$ and finally, a model that had constant values for both $\delta v$ and varied $\psi_{1}$. The constant laser parameters used in all cases were the value measured at the absorption line-centre. From the recovered measurement data it was clear that there is an induced error in the recovery of concentration and temperature if the variation of $\psi_{1}$ and $\delta v$ are not considered. However, the individual contributions to this error from $\psi_{1}$ and $\delta v$ vary as a function of the spectral line-shape and therefore

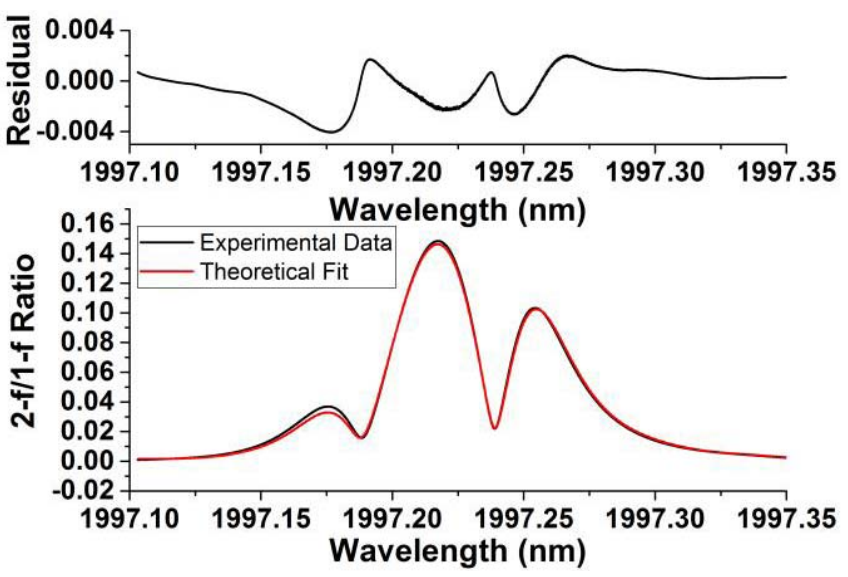

Fig. 8. High temperature 2f/1f experimental spectrum and comparison to model using the self-broadening coefficients and line-strengths values obtained from the high temperature spectroscopic characterization. Temperature $=$ $200{ }^{\circ} \mathrm{C}$, pressure $=1.023$ bar, path length $=5.5 \mathrm{~cm}$, concentration $=100 \%$ and modulation amplitude $=15 \mathrm{~mA}$.

with temperature and pressure. The recovered measurement error for both concentration and temperature for a $200^{\circ} \mathrm{C}$, atmospheric pressure simulation is approximately twice as large when the variation in $\delta v$ is considered than when the variation in $\psi_{1}$ is considered. More importantly, the overall error in the recovered concentration and temperature is lower if both $\psi_{1}$ and $\delta v$ are kept constant than if only one of these parameters are kept constant. The complex modelling required for the $2 \mathrm{f} / 1 \mathrm{f}$ technique, and the strong dependence on the laser parameters to the recovered line-shape implies that it is essential to fully characterize the laser over the full wavelength range. This full characterization ensures that the complexities of understanding where modelling errors will be introduced can be avoided.

\section{Aero-Engine Measurements}

The validated $2 \mathrm{f} / 1 \mathrm{f}$ measurement technique described in section III was then used to measure the integrated single path $\mathrm{CO}_{2}$ concentration and average temperature from the plume of an aero engine within a test-bed facility. The optical launch and receive optics were located at opposite ends of the testbed's detuner that is used to dampen and extract the exhaust of the engine. The diameter of the plume at the detuner had been previously measured to be $80 \mathrm{~cm}$ [20]. The output light from the laser is transmitted through 50 m of SMF 28 optical fiber from the control room to the detuner and launched across the detuner using a Thorlabs aspheric lens over a total path length of $\sim 200 \mathrm{~cm}$ and through the centre line of the engine plume. The transmitted beam is focussed onto a Thorlabs DET10 D/M extended InGaAs detector using a concave mirror. The output from the detector is transmitted down a $50 \mathrm{~m}$ length of coaxial cable to the data processing and recording electronics of the same form as shown in figure 3 . However, in this case a preamplifier is used for impedance matching the detector output with the LIA input and amplifying the signal from the photodiode.

Figures 9, 10 and 11 show the $R_{1 f}, R_{2 f}$ and the 2f/1f signals for a current modulation amplitude of $10 \mathrm{~mA}$ 


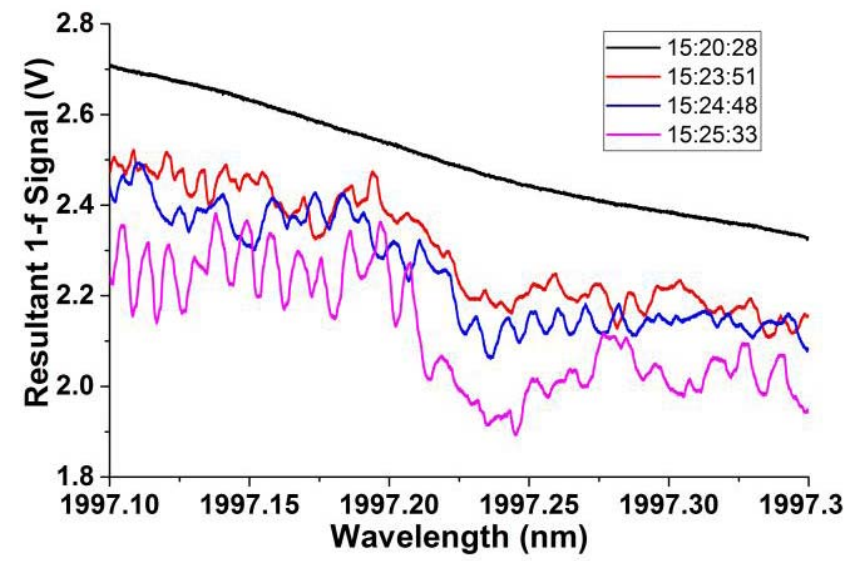

Fig. 9. $R_{1 f}$ signal corresponding to increasing thrust levels from engine start to full thrust, with the time stamp for the dataset shown in the legend. At $100 \%$ thrust the $\mathrm{CO}_{2}$ signal is clearly visible but there is a significant increase in noise levels.

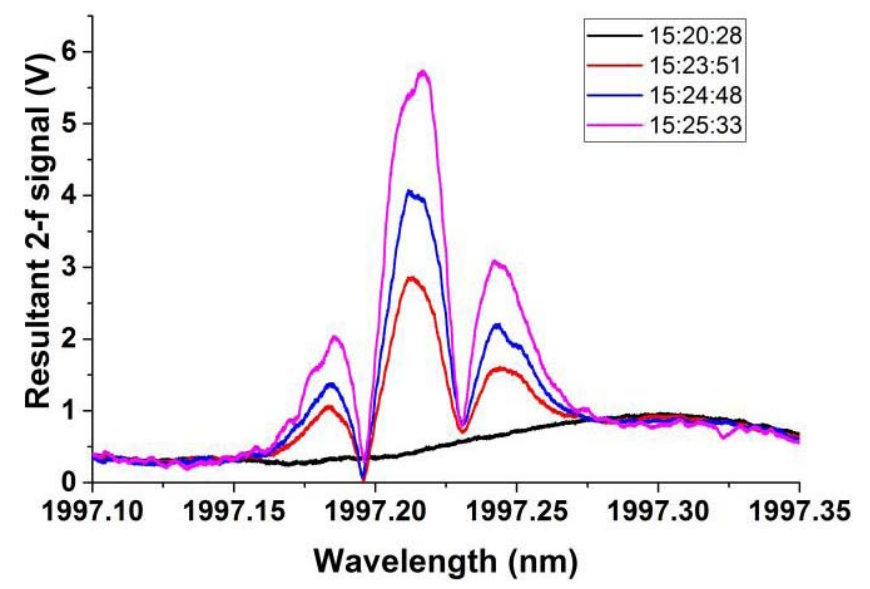

Fig. 10. $R_{2 f}$ signal corresponding to increasing thrust levels from engine start to full thrust, with the time stamp for the dataset shown in the legend.. The $\mathrm{CO}_{2}$ signal is visible for all thrust levels and the non-linear background is clearly evident

at a frequency of $200 \mathrm{kHz}$. Data is shown forthe engine static ( $0 \%$ Thrust) and for the engine operating at varying thrusts, up to maximum thrust (100\% Thrust). Figure 9 shows that the optical signal incident on the detector reduces during engine operation. As the overall optical power does not recover when the engine returns to the static situation this loss is assumed to be due to mechanical misalignment. It is also clear from Figure 9 that the overall optical noise increases as a function of engine thrust, due to mechanical vibration and optical beam steering effects of the plume turbulence. As the 2f/1f technique does not require normalization for optical intensity, the reduction in the total optical power does not affect the accuracy of signal recovery. Furthermore, the beam steering noise is effectively cancelled as the magnitude of $\mathrm{I}$ and $\Delta \mathrm{I}$ are both equally affected by the noise, as evident in Figure 11.

The highly non-linear background, $i_{2}$, is evident in Figure 10, showing a significant etalon arising from the wavelength dependence of the optical components used in the system. This highly non-linear background is normalised

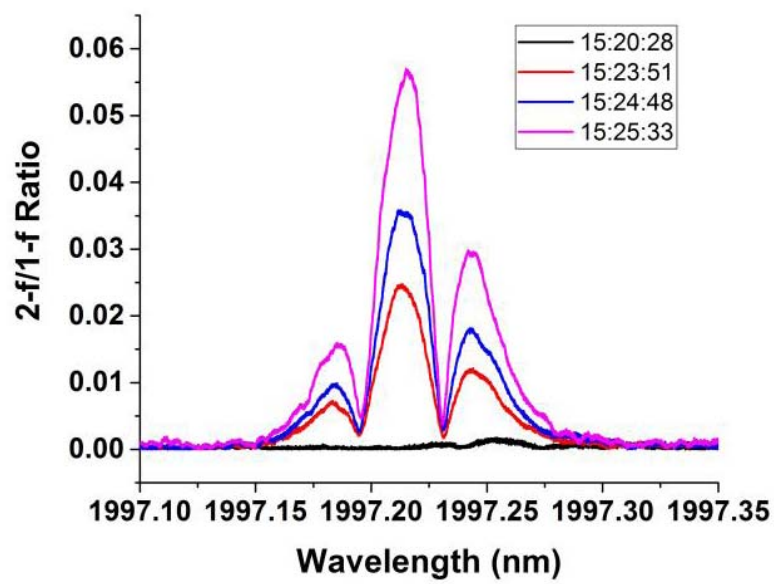

Fig. 11. The measured 2-f/1-f ratio with background subtraction for the same thrust levels as shown in figures 9 and 10. The time stamp for the dataset shown is in the legend and the recovered concentration and temperatures for these signals are shown in Figures 14 and 15.

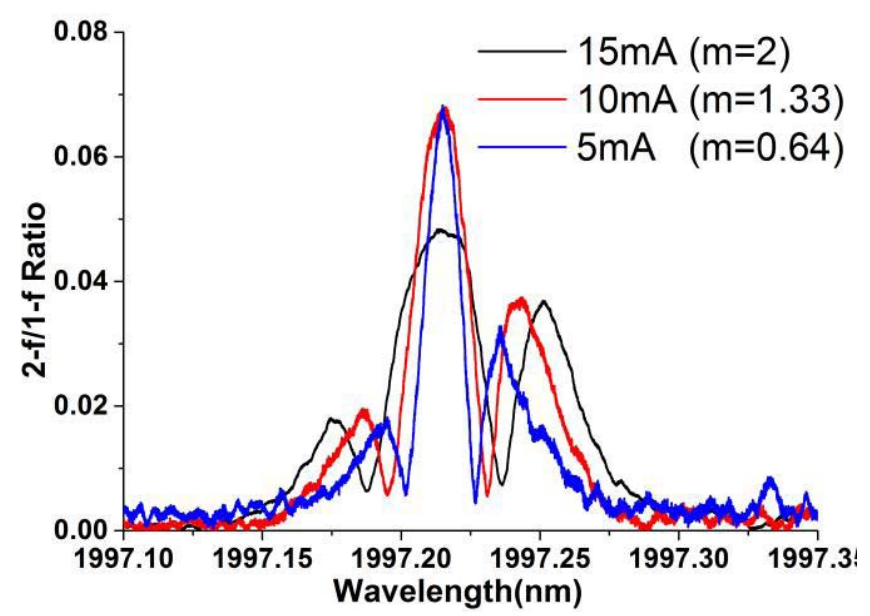

Fig. 12. 2f/1f signals recorded at varying modulation indices at the same engine operating conditions. Note the reduction in the overall noise as a function of modulation index and the reduced signal magnitude for $\mathrm{m}=2$.

by the magnitude of the $1 \mathrm{f}$ background signal, to compensate for any optical power variation, and then subtracted as described in equation (5).

Figure 12 shows $2 \mathrm{f} / 1 \mathrm{f}$ signals recovered when the aero engine was operating at constant thrust for current modulation amplitudes of 5,10 and $15 \mathrm{~mA}$, resulting in modulation indices of $0.64,1.33$ and 2 respectively. The theoretical $2 \mathrm{f} / 1 \mathrm{f}$ ratio at line centre is shown in Figure 13 as a function of modulation index for the same aero-engine operating conditions. As can be seen from Figures 12 and 13, the 2f/1f ratio is approximately equal for the $\mathrm{m}=0.64$ and 1.33 signals. This shows that there is good agreement between the theoretical m-values and the $\mathrm{m}$-values obtained during the LSQ fitting process for concentration and temperature. As the modulation index is increased there is clearly a reduction in the noise contribution, and the resulting signal to noise ratios for the current modulation amplitudes of 5, 10 and $15 \mathrm{~mA}$ are $18.75 \mathrm{~dB}, 21.9 \mathrm{~dB}$ and $21.7 \mathrm{~dB}$ respectively.

Figures 14 and 15 show the obtained concentration and temperature measurements from the exhaust plume as the 


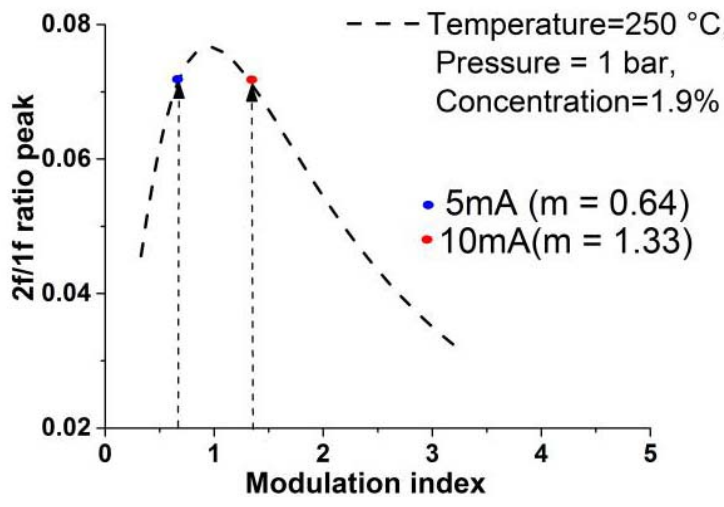

Fig. 13. The variation of the $R_{2 f} / R_{1 f}$ signal at line centre as a function of modulation index. The values of $\mathrm{m}$ corresponding to a current modulation amplitude of 5 and $10 \mathrm{~mA}$ at $250^{\circ} \mathrm{C}$ and 1 bar are shown to give equal output signals, agreeing with the data shown in figure 12 .

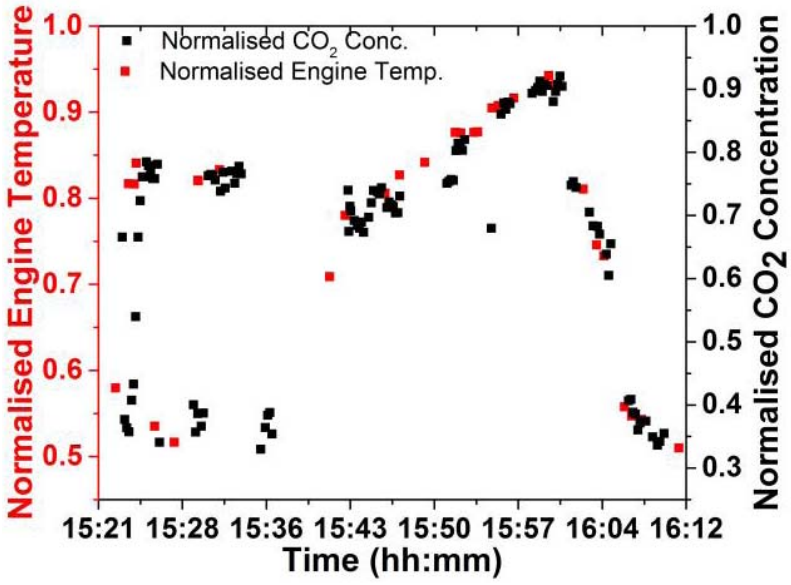

Fig. 14. The normalised concentration values measured in an aero engine exhaust plume using the $2 \mathrm{f} / 1 \mathrm{f}$ WMS technique and a comparison with the normalised core engine temperature.

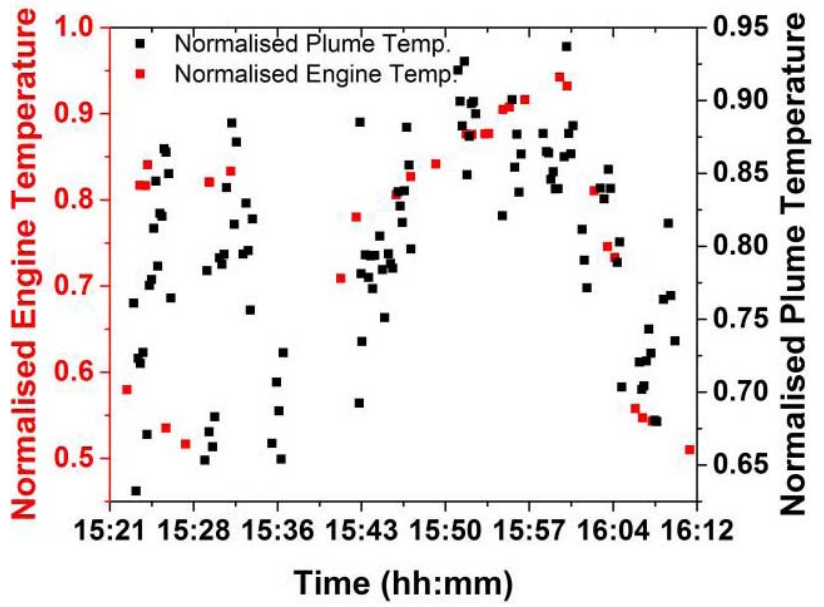

Fig. 15. A comparison of the normalised core engine temperature and the average temperature measured across the exhaust plume using the $2 \mathrm{f} / 1 \mathrm{f}$ WMS technique.

aero engine thrust is varied. The normalised temperature of the engine combustor is also shown in figs. 14 and 15 for comparison, and is used as a surrogate for engine thrust condition. An increase in engine thrust corresponds to an increase in core engine/combustor exit temperature and therefore an increase in the downstream plume temperature and the overall output of $\mathrm{CO}_{2}$. The recovered concentration and temperature measurements show excellent correlation to the measured temperature of the engine core.

Previous $\mathrm{CO}_{2}$ measurement campaigns using extractive gas sampling have shown that the expected $\mathrm{CO}_{2}$ output will range from $2 \%$ at idle, increasing to between 4 and $5 \%$ at full throttle. The maximum concentration measured in the downstream engine plume during this campaign of in situ sampling is $1.8 \%$ at full throttle. However, mixing of engine bypass air and entrained air around the engine prior to the measurement plane needs to be taken into account. This engine type has a by-pass ratio of $3.1: 1$, so a measured idle $\mathrm{CO}_{2}$ concentration of $\sim 0.68 \%$ results in an engine core output concentration of $\sim 2.78 \%$ when the by-pass air is considered. However, the predicted concentrations will be a slight overestimation of the actual engine core concentration without taking account of the flowrate of the entrained air passing around the engine (which will change depending on engine thrust condition). For this test campaign the entrained flowrate is not known.

No previous campaigns for the measurement of the plume temperature have been carried out. In this work, the maximum temperature across the detuner was measured to be $280^{\circ} \mathrm{C}$. As discussed for the concentration measurements above, the mixing of the exhaust gas with the by-pass and entrained air implies that this measured temperature will be significantly lower than the actual plume temperature. In this case, it is not possible to predict the effect of the mixed gas to estimate the plume temperature.

The standard deviation of concentration measurements and temperature was determined during a significant period of engine operation at constant conditions, resulting in values of $2.1 \%$ and $5 \%$ respectively. It is clear that the accuracy of the concentration measurement is better than the temperature measurement and a number of reasons for this have been considered. The sensitivity of the $2 \mathrm{f} / 1 \mathrm{f}$ signal to variations in temperature is around half of the sensitivity for concentration. Furthermore, variation in temperature affects both the integrated area of the recovered signal in the same manner as concentration, but also the width of the signal due to variation in the collisional broadening term resulting in a variation in the calculated m-value.

\section{Conclusions}

In this work, we have shown how the $2 \mathrm{f} / 1 \mathrm{f}$ technique can be used to accurately recovery the path integrated $\mathrm{CO}_{2}$ concentration and the average temperature from the exhaust plume of a commercial aero-engine. The necessary steps required to ensure that the technique is used in an appropriate manner are presented; i.e. the measurement of the temperature dependence of the spectral parameters of the target species, the full characterization of the laser characteristics and the determination of the non-linear optical signal in-situ. The presented methodology has been implemented with due recognition of further application in the FLITES tomographic system, and the considerations required for this campaign have been discussed in detail. 


\section{ACKNOWLEDGMENT}

The data for this paper is publicly available at the following DOI 10.15129/29f0b152-bc4a-4d78-92e9-d26ea94dc750.

\section{REFERENCES}

[1] P. Wright et al., "Progress towards non-intrusive optical measurement of gas turbine exhaust species distributions," in Proc. IEEE Aerosp. Conf., Mar. 2015, pp. 1-14.

[2] J. T. C. Liu, J. B. Jeffries, and R. K. Hanson, "Wavelength modulation absorption spectroscopy with $2 f$ detection using multiplexed diode lasers for rapid temperature measurements in gaseous flows," Appl. Phys. B, Lasers Opt., vol. 78, pp. 503-511, Feb. 2004.

[3] H. Li, G. B. Rieker, X. Liu, J. B. Jeffries, and R. K. Hanson, "Extension of wavelength-modulation spectroscopy to large modulation depth for diode laser absorption measurements in high-pressure gases," Appl. Opt., vol. 45, no. 5, pp. 1052-1061, 2006.

[4] T. Fernholz, H. Teichert, and V. Ebert, "Digital, phase-sensitive detection for in situ diode-laser spectroscopy under rapidly changing transmission conditions," Appl. Phys. B, Lasers Opt., vol. 75, nos. 2-3, pp. 229-236, 2002.

[5] G. Stewart, W. Johnstone, J. R. P. Bain, K. Ruxton, and K. Duffin, "Recovery of absolute gas absorption line shapes using tunable diode laser spectroscopy with wavelength modulation-Part I: Theoretical analysis," J. Lightw. Technol., vol. 29, no. 6, pp. 811-821, Mar. 15, 2011.

[6] J. R. P. Bain, W. Johnstone, K. Ruxton, G. Stewart, M. Lengden, and K. Duffin, "Recovery of absolute gas absorption line shapes using tunable diode laser spectroscopy with wavelength modulationPart 2: Experimental investigation," J. Lightw. Technol., vol. 29, no. 7, pp. 987-996, Apr. 1, 2011.

[7] G. B. Rieker, J. B. Jeffries, and R. K. Hanson, "Calibration-free wavelength-modulation spectroscopy for measurements of gas temperature and concentration in harsh environments," Appl. Opt., vol. 48, no. 29 , pp. $5546-5560,2007$.

[8] P. Wright et al., "Implementation of non-intrusive jet exhaust species distribution measurements within a test facility," in Proc. IEEE Aerosp. Conf., Mar. 2016, pp. 1-14.

[9] L. S. Rothman et al., "The HITRAN2012 molecular spectroscopic database," J. Quant. Spectrosc. Radiat. Transf., vol. 130, pp. 4-50, Nov. 2013.

[10] Y. Feng et al., "LD-seeded thulium-doped fibre amplifier for $\mathrm{CO}_{2}$ measurements at $2 \mu \mathrm{m}$," in Proc. Europhoton Conf., 2014, p. 1.

[11] N. Jacquinet-Husson et al., "The 2015 edition of the GEISA spectroscopic database," J. Mol. Spectrosc., vol. 327, pp. 31-72, Sep. 2016.

[12] S. A. Tashkun, V. I. Perevalov, J. L. Teffo, A. D. Bykov, and N. N. Lavrentieva, "CDSD-296, the carbon dioxide spectroscopic databank: Version for atmospheric applications," in Proc. 4th Symp. High Resolution Mol. Spectrosc., Krasnoyarsk, Russia, Jul. 2003. [Online]. Available: ftp://ftp.iao.ru/pub/CDSD-296/S

[13] C. Corsi, F. D'Amato, M. De Rosa, and G. Modugno, "High resolution measurements of line intensity, broadening and shift of $\mathrm{CO}_{2}$ around 2 m," Eur. Phys. J. D-Atomic, Mol., Opt. Plasma Phys., vol. 6, no. 3, pp. 327-332, 1999.

[14] R. M. Mihalcea, D. S. Baer, and R. K. Hanson, "Diode-laser absorption measurements of $\mathrm{CO}_{2}$ near $2.0 \mu \mathrm{m}$ at elevated temperatures," Appl. Opt., vol. 37, no. 36, pp. 8341-8347, 1998.

[15] M. E. Webber, S. Kim, S. T. Sanders, D. S. Baer, R. K. Hanson, and Y. Ikeda, "In situ measurements of $\mathrm{CO}_{2}$ by use of a distributed-feedback diode-laser sensor near $2.0 \mu \mathrm{m}$," Appl. Opt., vol. 40, no. 6, pp. 821-828, 2001.

[16] W. Johnstone, A. J. McGettrick, K. Duffin, A. Cheung, and G. Stewart, "Tunable diode laser spectroscopy for industrial process applications: System characterization in conventional and new approaches," IEEE Sensors J., vol. 8, no. 7, pp. 1079-1088, Jul. 2008.

[17] K. Sun, X. Chao, R. Sur, C. S. Goldenstein, J. B. Jeffries, and R. K. Hanson, "Analysis of calibration-free wavelength-scanned wavelength modulation spectroscopy for practical gas sensing using tunable diode lasers," Meas. Sci. Technol., vol. 24, p. 125203, Oct. 2013.

[18] A. Chighine et al., "TDLAS using FPGA-based lock-in detection for multi-channel chemical species tomography," in Proc. IEEE SENSORS, Nov. 2015, pp. 1-4.

[19] T. Benoy, M. Lengden, G. Stewart, and W. Johnstone, "Recovery of absorption line shapes with correction for the wavelength modulation characteristics of DFB lasers," IEEE Photon. J., vol. 8, no. 3, Jun. 2016, Art. no. 1501717.
[20] J. R. P. Bain, "Near infrared tunable diode laser spectroscopy for aero engine related applications," Ph.D. dissertation, Dept. Electron. Elect. Eng., Univ. Strathclyde, Glasgow, U.K., 2012.

Thomas Benoy received the M.Sc. (Hons.) degree in photonics from the Cochin University of Science and Technology, Kerala, India, and the Ph.D. degree in laser spectroscopy from the Centre for Microsystems and Photonics, University of Strathclyde, under the supervision of Prof. W. Johnstone and Dr. M. Lengden. His Ph.D. thesis was part of the FLITES Consortium. His research interests include precision spectroscopy and laser diagnostics.

Dr. T. Benoy is a member of the Optical Society of America and an Associate Member of the Institute of Physics.

David Wilson received the B.Sc. (Hons.) degree in mathematics and physics and the Ph.D. degree in physics, in 2008 and 2012, respectively. He studied the use of pulsed quantum cascade lasers in high resolution mid-infrared spectroscopy.

He worked as an instrumentation scientist at Cascade Technologies, Ltd., until late 2013 when he returned to the University of Strathclyde. He is currently a Research Associate with the Centre for Microsystems and Photonics where he is engaged in the developing spectroscopy techniques using semi-conductor diode lasers. His current research interests include tunable laser techniques for measurement of concentration and temperature in harsh environments, miniature photo-acoustic sensors, and non-dispersive infrared sensors for low cost environmental monitoring.

Dr. Wilson is a member of the Institute of Physics.

Michael Lengden received the M.Phys. degree in physics and the Ph.D. degree from the University of Manchester, Manchester, U.K., in 2001 and 2006, respectively, where he was engaged in the stepwise excitation of atomic and molecular metastable states.

He was an Applications Engineer with the Laboratory Impex Systems Ltd., from 2006 to 2007 and a Research Fellow at the Centre for Microsystems and Photonics from 2007 to 2010. He is currently a Senior Lecturer with the Electronics and Electrical Engineering Department, University of Strathclyde, Glasgow, U.K., where he is engaged in high-temperature and high-pressure gas composition measurements using a tunable diode laser spectroscopy. His current research interests include applied tunable diode laser spectroscopy techniques in harsh environments and miniaturized photoacoustic sensor development.

Dr. Lengden is a member of the Institute of Physics.

Ian Armstrong received the B.Eng. (First Class Hons.) degree in electronic and electrical engineering from the University of Strathclyde, Glasgow, U.K., in 2001, and the Eng.D. degree from the University of Strathclyde in 2006, for his research in semiconductor optical amplifier integration, in collaboration with Kamelian Ltd. He worked until 2013 as a Research Fellow at the Centre for Microsystems and Photonics, University of Strathclyde, developing TDLS-based gas detection systems in the near- and mid-IR spectral regions. Currently, he is the Senior Photonics Engineer with OptoSci Ltd., bringing TDLS technology to industrial monitoring applications.

George Stewart received B.Sc. (Hons.) and Ph.D. degrees in integrated optics from the University of Glasgow, U.K., in 1974 and 1979, respectively.

He continued research on integrated optics at the University of Glasgow until 1985, and then joined the University of Strathclyde, Glasgow, where his research has been in the areas of fiber optic components, evanescent wave devices, optical sensors, fiber lasers, and DFB laser spectroscopy. He has authored or co-authored over 180 technical and scientific papers in photonics.

Prof. Stewart is a member of the Optical Society of America and the Institute of Physics.

Walter Johnstone received the B.Sc. (Hons.) degree in chemical and material sciences and the Ph.D. degree in laser applications from the University of Strathclyde, Glasgow, U.K., in 1977 and 1982, respectively.

$\mathrm{He}$ is currently a Research Professor of Photonic Systems in electronic and electrical engineering with Strathclyde University and has served in senior management positions as the Head of Department and the Vice Dean (Research) at the Faculty of Engineering. Prior to his academic career, he held project engineering and senior technical management positions at Pilkington Optronics Ltd., (now Thales) and Logitech Ltd. Since 1994, he has been a Director of OptoSci Ltd., Glasgow, where he has been instrumental in bringing a number of photonics products to market. His research work now focuses on high-resolution industrial spectroscopy for gas measurement applications in harsh environments, such as gas turbine (aero) engines and fuel cells. This work and previous research in optical waveguide components, fiber lasers, and distributed fiber sensing have led to over 200 technical publications. 